\title{
GLASS TRANSITION IN RUBBERY MATERIALS
}

\author{
C. MiCHAEL ROLAND* \\ NAVAl RESEARCh LABORATORY, CHEMISTRy Division, CODE 6120, WASHINGTON, DC 20375-5342
}

RUBBER CHEMISTRY AND TECHNOLOGY, Vol. 85, No. 3, pp. 313-326 (2012)

\begin{abstract}
When the perturbation frequency imposed on a rubber falls within the glass transition zone of its viscoelastic spectrum, energy absorption is maximized. This phenomenon is the operative mechanism for various applications of elastomers requiring large energy dissipation. Nevertheless, a fundamental understanding of the glass transition is lacking. The diversity of properties that depend both on chemical structure and thermodynamic conditions makes modeling difficult and a first principles theory perhaps unachievable; indeed, the number of models for the glass transition seems to be inversely proportional to their ability to accurately describe the myriad behaviors. The progress made at quantifying the role of the thermodynamic variables temperature, $T$, and density, $\rho$, on the dynamics is described. An important aspect of the work was the discovery that relaxation times and viscosities of molecular liquids and polymers superpose when plotted against the scaling variable $T / \rho^{\gamma}$, with the scaling exponent $\gamma$ a material constant sensibly related to the nature of the intermolecular repulsive potential; thus, dynamic spectroscopy measurements can be used to quantify the forces between molecules. Other properties derive from the scaling behavior, including the Boyer-Spencer rule and the correlation of fluctuations in the potential energy with fluctuations in the virial pressure. [doi:10.5254/rct.12.87987]
\end{abstract}

\section{CONTENTS}

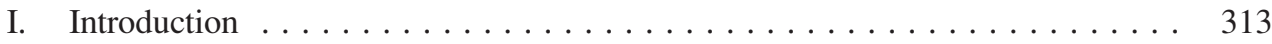

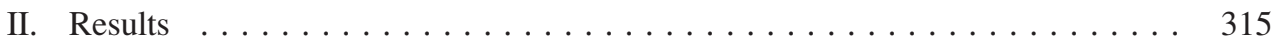

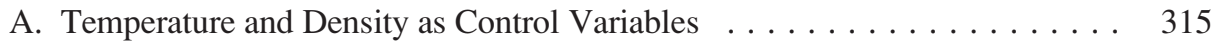

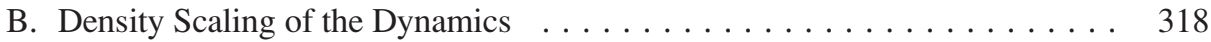

C. Fluctuations of the Potential Energy and the Virial . . . . . . . . . . . . . 322

D. Comparison to Time-Temperature Superpositioning . . . . . . . . . . . . 322

E. Boyer-Spencer Rule . . . . . . . . . . . . . . . . . . . . . 323

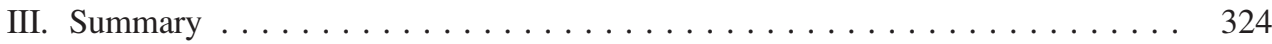

IV. Acknowledgements . . . . . . . . . . . . . . . . . . 324

V. References .......................... 324

\section{INTRODUCTION}

The behavior of polymers when vitrification is imminent has relevance to a number of applications. An elastomer deformed at sufficiently high rates, typically beyond $10^{4} \mathrm{~s}^{-1}$ depending on the glass transition temperature, $T_{g}$, responds in a "leathery" fashion as it enters the glass transition zone of the viscoelastic spectrum. Energy dissipation of polymers is largest in the transition zone, and applications of elastomers can exploit this strain-induced, reversible phase change. One such technology is improved wet skid resistance of tires. The tread rubber is deformed by surface asperities, and on wet or rough surfaces, this mechanism dominates the apparent friction of the sliding tire. ${ }^{1-3}$ An optimal wet-skidresistant rubber dissipates substantial energy by responding within the viscoelastic glass transition zone to maximize mechanical hysteresis. The frequency associated with wet skidding is in the range from $10^{3}$ to $10^{6} \mathrm{~Hz},{ }^{4,5}$ so tread rubbers have high glass transition temperatures, so that at ambient temperatures, high frequency deformations induce a transition to the glassy state. ${ }^{6,7}$ 
Another application of rubber that relies on vitrification is sound attenuation, ${ }^{8}$ with the greatest absorption achieved if the elastomer undergoes its rubber-to-glass transition at the sonic frequency. For this reason, the selection of damping materials for sound attenuation, such as suppression of sonar reflections by submarines, is governed primarily by their $T_{g}$, with the objective of having the loss tangent peak fall within the sound frequencies at the relevant temperature. Rubber blends, polyurethanes, and polyureas are often used in these applications because their broad transitions make the acoustic response less sensitive to temperature. ${ }^{8,9}$

Nature also uses the glass transition phenomenon to dissipate energy. Under physiological conditions, the protein elastin comprising arterial walls begins to transition to the glassy state at frequencies beyond $5 \mathrm{~Hz}$ (that is, somewhat faster than the heart rate of the mammal, the exact value being species dependent). Thus, although normally the elastin enables arteries to elastically contract during the diastolic phase of the blood pressure cycle, at higher rates the arterial walls absorb energy. This mechanical dissipation serves the purpose of suppressing high frequency perturbations induced by turbulent flow of blood around obstacles such as plaque. ${ }^{10}$

A recent development in military technology exploits the impact-induced glass transition of elastomeric coatings applied to the front surface of armor in order to mitigate ballistic and explosive assault. ${ }^{11-13}$ The strain rate for bullets and bomb fragments striking a coating over a hard surface, estimated as the ratio of the projectile speed to the coating thickness, is about $10^{5} \mathrm{~s}^{-1}$. Thus, elastomers with sufficiently high $T_{g}$ will undergo the glass transition upon impact, with consequent conversion of the projectile's kinetic energy to heat. The transient hardening of the polymer can also contribute to mitigating the effect of the impact.

Developing applications that take full advantage of the properties of rubber when the segmental dynamics are dominant requires a theory or predictive model. This task is complicated by the wealth of properties and diverse phenomena exhibited by materials approaching vitrification. ${ }^{14,15}$ Many experimental techniques have been brought to bear on this problem, with dielectric spectroscopy and viscosity measurements in particular providing a large amount of data covering broad ranges of frequency and thermodynamic conditions (e.g., temperature and pressure). An intriguing property that remains poorly understood despite much research is the cause of the spectacular slowing down of the dynamics near the glassy state. The viscosity, $\eta$, and reorientational correlation time of molecular liquids or local segmental relaxation time of polymers, $\tau$, increase by many orders of magnitude for small decreases of temperature; the effect corresponds to apparent activation energies 10 -fold larger than that for common chemical reactions. At the glass

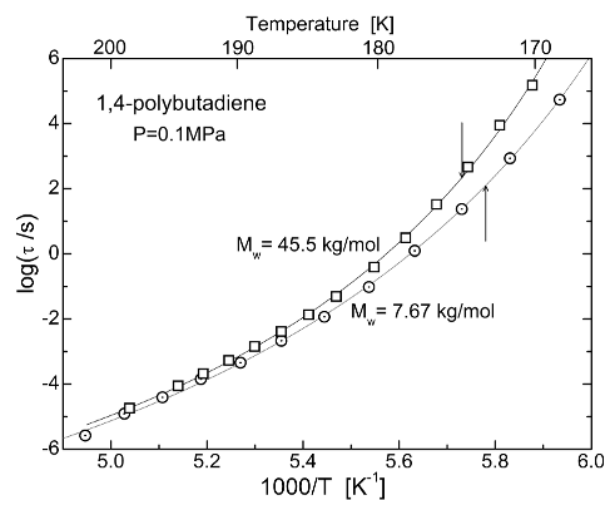

FIG. 1. - Local segmental relaxation times measured dielectrically for two polybutadienes having the indicated molecular weights. The dynamics change by more than five orders of magnitude over a $30^{\circ}$ temperature range. The arrows denote $T_{g}$ measured from the change in thermal expansivity. 
transition temperature the relaxation time, $\tau$, becomes larger than the duration of any feasible experiment; that is, the material attains the glassy state. An operative definition of $T_{g}$ is the temperature at which $\tau=10^{3} \mathrm{~s}$, which is on the order of the time constant associated with calorimetry experiments (the transition temperature divided by the heating rate defines a time scale for thermal analysis measurements). Strongly non-Arrhenius dynamics are exhibited by all supercooled liquids and polymers, with representative data shown for polybutadiene in Figure $1 .^{16}$ (The term supercooling traditionally refers to liquids quenched below their crystallization temperature; however, more generally it is used to describe materials, even those incapable of crystallizing, that are close to their glass transition temperature.)

\section{RESULTS}

\section{A. TEMPERATURE AND DENSITY AS CONTROL VARIABLES}

The slowing down of molecular and segmental motions as temperature is reduced has two obvious causes: (i) The molecules lose thermal energy, reducing the rate at which local potential barriers to positional changes are overcome, as described by energy landscape models. ${ }^{17-19}$ (ii) The volume contraction on cooling increases molecular congestion, with vitrification a jamming phenomenon, interpreted using free volume concepts. ${ }^{20-24}$ Of course, these two effects are not mutually exclusive. In the supercooled regimen, molecular and segmental motions can be envisioned as infrequent jumps over potential energy barriers having heights sensitive to the arrangement of neighboring species.

Isobaric Arrhenius plots such as Figure 1 are not sufficient to resolve the influences of temperature and density on the dynamics. The effect of volume changes must be isolated to quantify their effect relative to that of thermal energy. This requires measurements as a function of pressure, with the equation of state then used to convert the pressure dependence of $\tau$ or the viscosity to a dependence on density. This procedure is illustrated with data for polyisoprene (Figure 2), the local

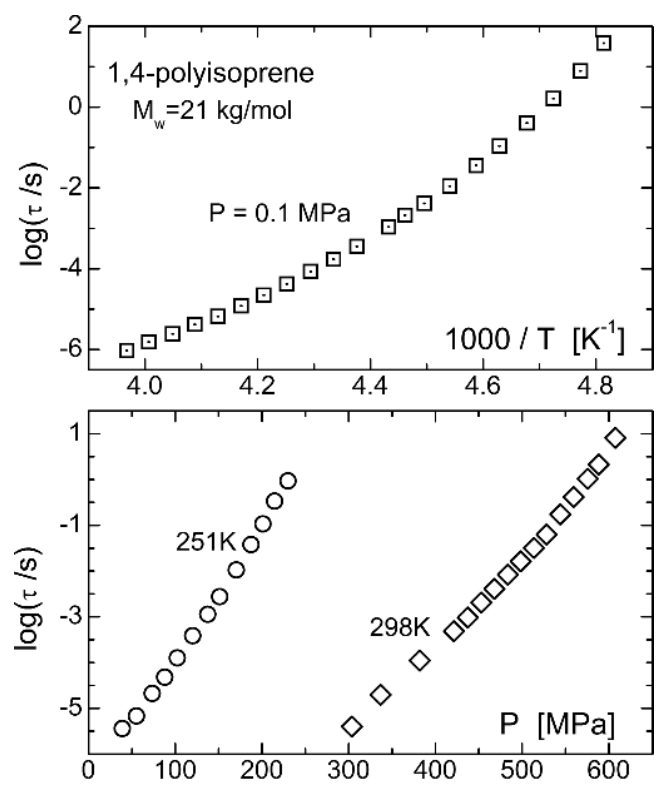

FIG. 2. - Local segmental relaxation times for polyisoprene (upper panel) as a function of inverse temperature at ambient pressure and (lower panel) versus pressure at various temperatures. 


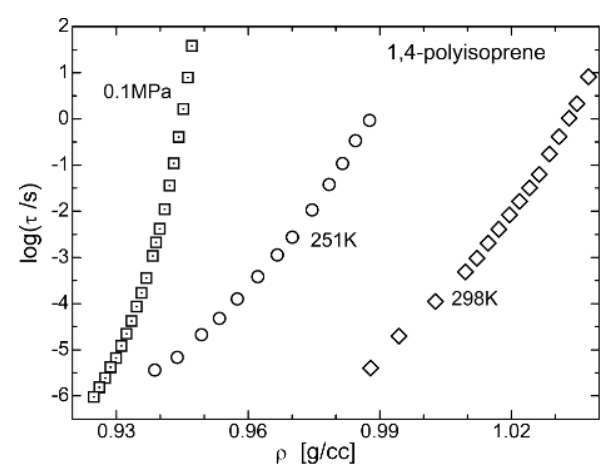

FIG. 3. - Local segmental relaxation times from Figure 2, plotted versus density at constant pressure with varying temperature (squares) and at fixed temperature with varying pressure (circles and diamonds).

segmental relaxation times having been determined from isobaric and isothermal dielectric measurements. ${ }^{25}$ Using the equation of state for this polymer, ${ }^{26}$

$$
\begin{aligned}
\rho^{-1}= & \left(1.094+6.29 \times 10^{-4} T\right. \\
& \left.+6.23 \times 10^{-7} T^{2}\right)\left[1-0.0894 \ln \left(1+\frac{P}{202 \exp \left[-4.65 \times 10^{-3} T\right]}\right)\right]
\end{aligned}
$$

(in which $T$ is in Celsius by convention and $P$ in MPa), the $\tau(\rho)$ in Figure 3 are calculated. ${ }^{25}$ These data demonstrate two points, that the relaxation times depend on density, because $\tau$ changes with pressure at constant temperature, and that thermal energy also exerts an effect, as evidenced by the steeper change in $\tau(\rho)$ when temperature is varied at constant pressure.

From these results, the relative influence of temperature and density on the dynamics is quantified from the ratio of the isochoric and isobaric activation energies ${ }^{14,27}$

$$
\begin{aligned}
& E_{\rho}(T, \rho)=\left.R \frac{\partial \ln \tau}{\partial T^{-1}}\right|_{\rho} \\
& E_{P}(T, P)=\left.R \frac{\partial \ln \tau}{\partial T^{-1}}\right|_{P}
\end{aligned}
$$

If $E_{\rho} / E_{P}=0$, there is no change in $\tau$ when density is maintained constant, indicating density is the control parameter. If $E_{\rho} / E_{P}=1$, the effect of temperature is the same whether or not the volume changes; that is, the dynamics are dominated entirely by thermal effects. From Figures 2 and 3, we obtain $E_{\mathrm{\rho}} / E_{P}=0.69 \pm 0.01$ at $T_{g}$ and $0.1 \mathrm{MPa}$, which is consistent with the value reported for natural rubber. ${ }^{28}$ Neither of the limiting behaviors of density- or temperature-dominated dynamics apply to polyisoprene, although because $E_{\rho} / E_{P}>0.5$, thermal energy exerts a stronger effect than does $\rho$.

Results for a large number of van der Waals materials are collected in Table I (molecular liquids) and Table II (polymers) ${ }^{14,29}$; hydrogen-bonded materials are excluded from these compilations. The tabulated values are for $T=T_{g}$; at higher temperatures, $E_{\mathrm{\rho}} / E_{P}$ decreases, ${ }^{30}$ indicating a stronger role of density as the dynamics becomes faster. Generally $E_{\rho} / E_{P}$ for small molecule substances is close to 0.5 ; that is, density and temperature exert a comparable influence on $\tau(T)$. However, for polymers, this activation energy ratio is significantly larger. In fact, only for poly (phenylene oxide), a peculiar polymer having a very high $T_{g}$ despite a flexible backbone, is $E_{\mathrm{\rho}} /$ $E_{P}$ less than 0.5. 
TABLE I

GLASS Transition TEMPERATURES, ACTIVATION ENERGy RATIOS, AND SCALING EXPONENTS FOR UNASSOCIATED MOLECULAR LIQUIDS

\begin{tabular}{lccc}
\hline Liquid & $T_{g}$ & $E_{\mathrm{\rho}} / E_{P}$ & $\gamma$ \\
\hline 62\% chlorinated biphenyl & 274 & 0.38 & 8.5 \\
1,1'-bis(p-methoxyphenyl)cyclohexane & 243 & 0.39 & 7 \\
1,1'-di(4-methoxy-5-methylphenyl)cyclohexane (BMMPC) & 263 & 0.41 & 8.5 \\
Cresolphthalein-dimethylether & 313 & 0.49 & 4.5 \\
54\% chlorinated biphenyl & 252 & 0.50 & 6.7 \\
Phenylphthalein-dimethylether & 249 & 0.53 & 4.5 \\
Ortho-terphenyl & 244 & 0.55 & 4 \\
42\% chlorinated biphenyl & 225 & 0.56 & 5.2 \\
Propylene carbonate & 159 & 0.64 & 3.7 \\
a-tocopherol acetate & 226 & 0.66 & 3.9 \\
\hline
\end{tabular}

The weaker influence that density exerts on the polymer dynamics is due to the plethora of intramolecular contacts. Interactions between covalent bonded segments are relatively insensitive to density because chemical bond lengths are resistant to change; thus, the effect of pressure is to increase interpenetration of the chain coils. (The same effect underlies the near pressure invariance of the dielectric strength of the normal mode of polymers, which is proportional to the end-to-end

TABLE II

Glass Transition TEMPERATURES, ACtivation EnERgy RATIOS, AND SCALING EXPONENTS FOR POLYMERS

\begin{tabular}{lccr}
\hline Polymer & $T_{g}(\mathrm{~K})$ & $E_{\mathrm{\rho}} / E_{P}$ & $\gamma$ \\
\hline Poly(phenylene oxide) & 462 & 0.25 & - \\
Polymethylphenylsiloxane & 248 & 0.52 & 5.6 \\
Polymethyltolylsiloxane & 261 & 0.55 & 5.0 \\
Polyvinylacetate & 302 & 0.6 & 2.6 \\
Diglycidylether of bisphenol A & 413 & 0.6 & 2.8 \\
Poly(phenol glycidyl ether)-co-formaldehyde & 258 & 0.63 & 3.5 \\
Polystyrene & 373 & 0.64 & 2.5 \\
Poly[(o-cresyl glycidyl ether)-co-formaldehyde] & 285 & 0.65 & 3.3 \\
Polypropylene glycol & 210 & 0.67 & 2.5 \\
Polyvinylmethylether & 250 & 0.69 & 2.9 \\
Polyvinylethylene & 253 & 0.70 & 1.9 \\
Polycyclohexylmethacrylate & 349 & 0.72 & 2.5 \\
Poly- $\alpha$-methylstyrene & 341 & 0.56 & 2.7 \\
Poly(2-vinylpyridine) & 337 & 0.72 & - \\
Polymethylmethacrylate & 380 & 0.74 & 1.8 \\
Polyoxybutylene & 199 & 0.75 & 2.7 \\
1,4-polyisoprene & 201 & 0.76 & 3.0 \\
Poly-2-chlorostyrene & 399 & 0.61 & 2.6 \\
Polyvinylphenol & 473 & 0.77 & - \\
Poly(4-vinylpyridine) & 399 & 0.77 & - \\
Polyvinylethylether & 241 & 0.81 & - \\
\hline & & &
\end{tabular}




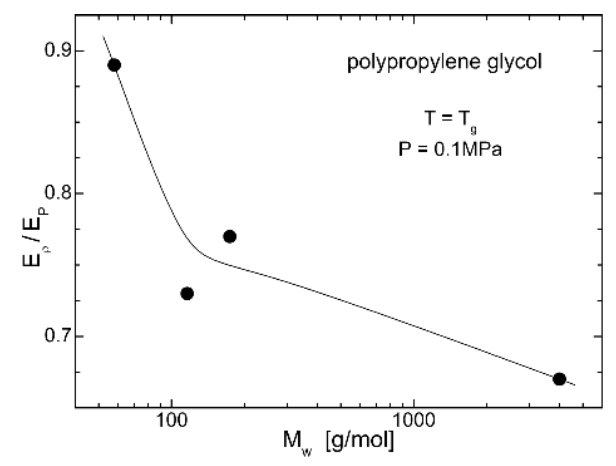

FIG. 4. - Activation energy ratio for polypropylene glycol versus molecular weight. With decreasing number of chain ends, the hydrogen bond concentration decreases, which enhances the effect density has on the segmental dynamics.

distance of chains. ${ }^{31,32}$ ) The fact that $\tau(T)$ for polymers is governed more by temperature than volume is ironic, given the historic attraction of free volume models in analyses of polymer viscoelastic properties. $^{21}$

Hydrogen-bonded substances behave differently than van der Waals materials, ${ }^{33}$ with the dynamics for the former invariably associated with large values of $E_{\rho} / E_{P}$. This dominance of temperature is because of thermal dissociation of $\mathrm{H}$-bonds that accompanies the raising of temperature, which accelerates the dynamics. Pressure may also reduce hydrogen bonding by making less empty volume available for the directional interactions. ${ }^{34-36}$ This reduction speeds up motions, countervailing the direct effect of pressure. The net effect is thermally dominated dynamics in associated liquids. Figure 4 shows results for polypropylene glycol, in which only the terminal carbons have hydroxyl groups, so that as the chain length increases, the extent of $\mathrm{H}$ bonding decreases. This reduction in hydrogen bonding with molecular weight gives rise to smaller values of $E_{\rho} / E_{P}$.

\section{B. DENSITY SCALING OF THE DYNAMICS}

The foregoing describes quantifying the relative contribution of temperature and density to the change in relaxation times or viscosity as a liquid or polymer is cooled toward $T_{g}$; however, that is not an explanation for the cause of the spectacular slowing down of the dynamics. Why does a $10 \%$ change in temperature change the rate of segmental motions by 10 orders of magnitude? This remains the primary issue in solving the problem of the glass transition. Some insight can be gained by examining how chemical structure affects the dynamics, and for this we employ density scaling. Although the density scaling of relaxation data was an empirical discovery, ${ }^{37}$ its origins trace to early studies of the nature of the intermolecular potential. ${ }^{38-41}$ To show this connection, we examine results from molecular dynamics simulations (mds). ${ }^{42}$

Computer simulations of glass formers often employ the Lennard-Jones 6-12 (LJ) potential (Figure 5), ${ }^{43}$ in which the potential energy is the sum of independent, spherically symmetric, twobody interactions among the particles

$$
U(r)=4 \varepsilon_{0}\left[(\sigma / r)^{12}-(\sigma / r)^{6}\right]
$$

where $\varepsilon_{0}$ is the depth of the potential well and $r$ the separation between the particles having size $\sigma$. The exponent of 6 in the attraction term describes van der Waals dispersion interactions, the product $r^{6} U(r)$ being proportional to the Hamaker constant. ${ }^{44}$ Repulsive forces are shorter ranged in 


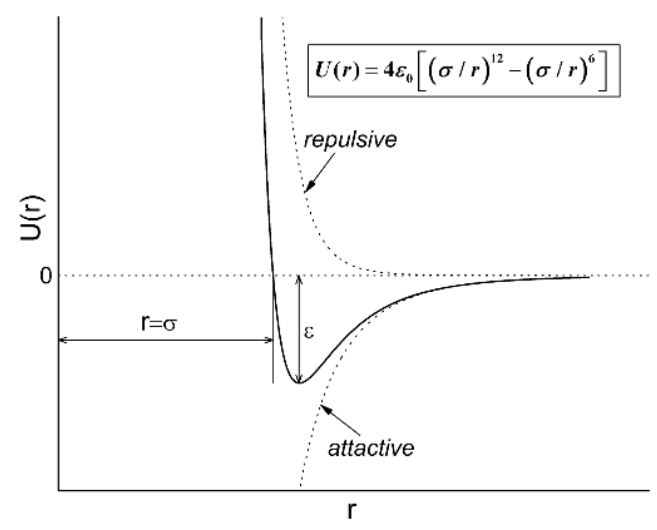

FIG. 5. - Lennard-Jones potential (Eq. 4). Note that the total potential is steeper for small interparticle distances than the repulsive term alone because of the contribution of the attractive component.

comparison to the attractions, and historically a value of 12 was assumed for the exponent on the repulsive term because of the computational convenience of simply squaring the value for the attractions. In reality, the exponent varies among materials, ${ }^{45,46}$ so that a more generally valid form of Eq. 4 is

$$
U(r)=4 \varepsilon_{0}\left[(\sigma / r)^{m}-(\sigma / r)^{6}\right]
$$

with $m$ related to the steepness of the intermolecular repulsive interaction for the particular material.

The longer range attractive forces in a liquid tend to cancel one another, so that for local properties, the molecular attractions can be approximated as a spatially uniform background potential. This implies that the repulsive interactions, strongest over short distances, dominate the local properties. ${ }^{47-49}$ The intermolecular potential can be simplified to an inverse power law $(\text { IPL) })^{38,39,41}$

$$
U(r)=\varepsilon_{0}(\sigma / r)^{m_{\mathrm{IPL}}}
$$

without explicit consideration of attractive forces. Alternatively, an extended IPL can be used, in which the attractive forces, which are necessary to cohere the liquid, are relegated to a background term that varies linearly with distance ${ }^{50}$

$$
U(r)=\varepsilon_{0}(\sigma / r)^{m_{e P L}}+\varepsilon_{1} r+\varepsilon_{2}
$$

where $\varepsilon_{1}$ and $\varepsilon_{1}$ are constants. Although the potentials in Eqs. 6 and 7 are approximations, their form is consistent with the fact that for liquids, the static structure factor at intermediate and large wave vectors is sensitive only to the repulsive part of the potential. ${ }^{51,52}$ That is, the local liquid structure is determined mainly by steric (packing) effects, governed by the repulsive forces. ${ }^{40,53}$

The great appeal of the IPL approximation is that when the potential has this form, or even approximately as in Eq. 7, thermodynamic properties such as energy, volume, and entropy ${ }^{40,41}$ and dynamic properties such as $\tau$ and the viscosity ${ }^{54,55}$ depend only on the scaled variable $\operatorname{Tr}^{m}$, or in terms of the experimentally measurable density, $T / \rho^{m / 3}$. This means that structural relaxation times for liquids and the local segmental relaxation times of polymers should conform to the scaling law ${ }^{37}$

$$
\tau=f\left(T / \rho^{\gamma}\right)
$$

in which $f$ is a function that is unknown a priori, with the scaling exponent $\gamma$ a material constant equal to $m / 3$. This scaling property, Eq. 8, is illustrated in Figure 6 for molecular liquids and Figure 7 


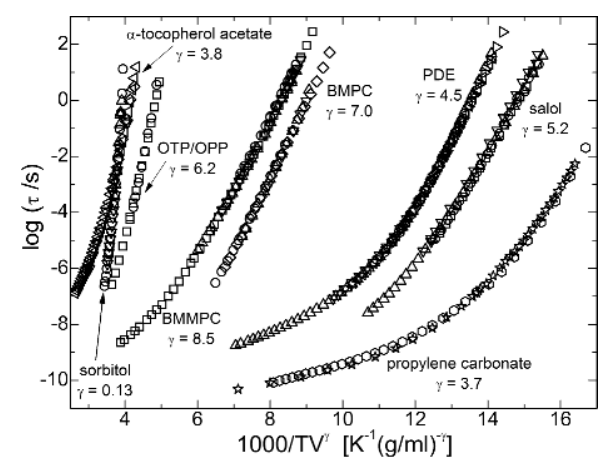

FIG. 6. - Scaling of molecular liquids. OTP/OPP: blend of 67\% o-terphenyl and 33\% o-phenylphenol; BMPC: 1, $1^{\prime}$-bis $(p$ methoxyphenyl)cyclohexane; BMMPC: 1,1'-di(4-methoxy-5-methylphenyl)cyclohexane; PDE:

phenolphthaleindimethylether; KDE: cresolphthalein-dimethylether. The scaling exponents are as indicated.

for polymers. (Strictly speaking, density scaling derived from an IPL potential applies only to reduced units; that is, the excess quantity above that for an ideal gas. However, for state points in the supercooled regime, the difference in scaling using reduced or unreduced units is negligible. ${ }^{56}$ ) Conformance to Eq. 8 has been demonstrated for hundreds of materials, ${ }^{14,15,57}$ limited only by the availability of the equation state and relaxation measurements as a function of both $T$ and $P$. Because rubbers have low glass transition temperatures, obtaining $\tau(P), \eta(P)$, or $\rho(T, P)$ near $T_{g}$ is difficult; hence, density scaling has been applied to only a few rubbers, for example, polyisoprene, 1,2-polybutadiene, and two siloxane polymers, and oligomeric materials such as polypropylene glycol and polyvinylmethylether. However, the expectation is that Eq. 8 should be valid for all nonassociated liquids and polymers.

The only known deviation from density scaling is for hydrogen-bonded materials. ${ }^{33}$ This is due to the changes in H-bond concentration, with consequent effect on the dynamics, with changes in temperature and (to a lesser extent) pressure. Thermal dissociation of hydrogen bonds enhances molecular mobility, an effect unaccounted for by the scaling. Also, higher pressures or densities, by reducing the available volume, may cause directional bonds to be sacrificed in order to facilitate

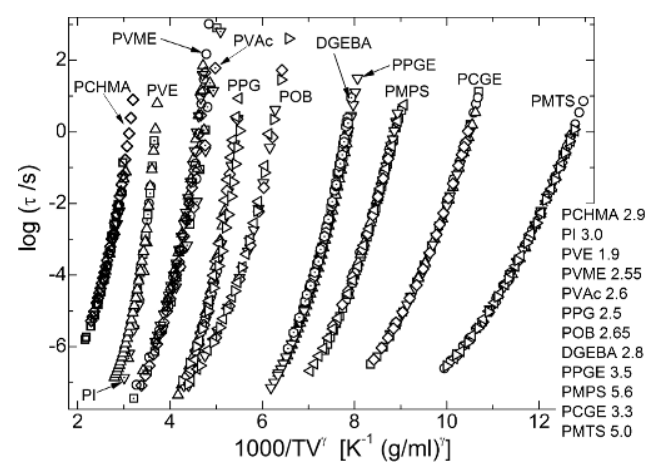

FIG. 7. - Scaling of polymers. PCHMA: polycyclohexylmethacrylate; PI: 1,4-polyisoprene; PVE: polyvinylethylene; PVME: polyvinylmethylether; PVAc: polyvinylacetate; PPG: polypropylene glycol; POB: polyoxybutylene; DGEBA: diglycidyl ether of bisphenol A; PPGE: poly[(phenyl glycidy ether)-co-formaldehyde]; PMPS: polymethylphenylsiloxane; PCGE: poly[(o-cresyl glycidyl ether)-co-formaldehyde]; PMTS: polytmethyltolylsiloxane. The scaling exponents are as indicated. 

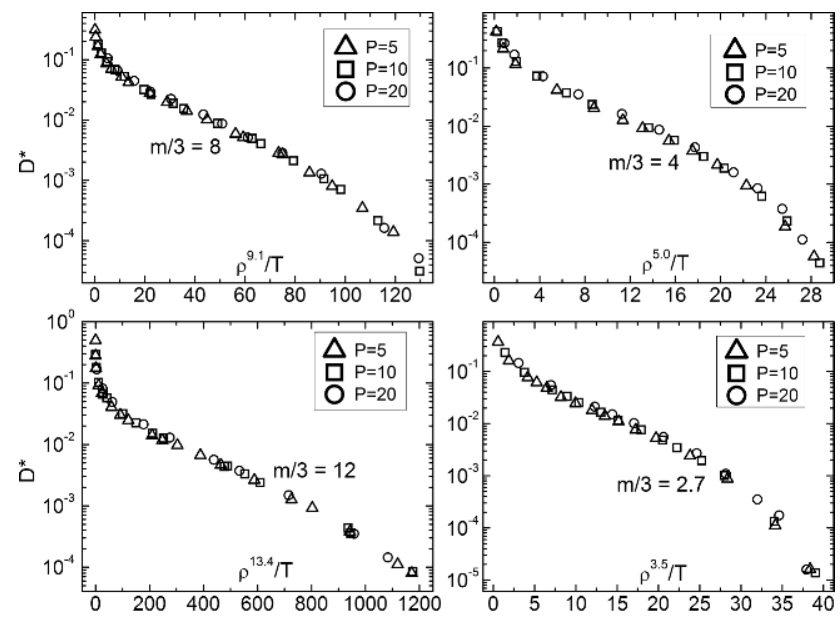

FIG. 8. - Scaling of the diffusion constants for LJ particles having the indicated exponent for the repulsive term in Eq. 5. Pressures are in LJ units, and the quantity plotted on the ordinate is the reduced $D^{*}=\left(\rho^{1 / 3} / T^{1 / 2}\right) \mathrm{D}$ (see ref. 56).

molecular packing. These two effects, absent in nonassociated liquids, cause a breakdown of the otherwise general conformance to density scaling.

The scaling exponent for a given material is determined empirically; that is, $\gamma$ is obtained as the value that superposes dynamic data $\left(\tau, \eta\right.$, or time-temperature shift factors, $\left.a_{T}\right)$. However, we expect $\gamma$ to reflect the steepness of the intermolecular repulsive potential. This statement is strictly correct for an IPL and valid for real materials if the IPL is an accurate approximation of the local intermolecular potential. To show this, density scaling was applied to diffusion constants from $\mathrm{mds}$, in which different values were used for the repulsive exponent in Eq. $5 .{ }^{42}$ The scaling of the data is shown in Figure 8, with the obtained scaling exponents compared in Table III to the corresponding values of $m$.

There is consistency between the two quantities; however, for all cases $\gamma>m / 3$. This discrepancy between the value that superposes the diffusion constants and that expected from $U(r)$ arises because of the influence of the attractive term in Eq. 5. The effective potential is steeper in the region of $r$ close to the minimum than the repulsive term alone (see Figure 5); thus, in the region of particle distances relevant to the dynamic data in Figure 7, $m$ underestimates the steepness. ${ }^{42}$ To quantify this, we fit $U(r)$ to a power law from the distance of closest approach between particles, at which the radial distribution function becomes nonzero, to the value of $r$ corresponding to the halfheight of the first peak in this function. This comprises the range of interparticle separations over which the particles spend most of their time in the supercooled state. The fitted power law defines an effective steepness for the repulsive potential, $m_{\mathrm{eff}}=\frac{d \log U(r)}{d \log r}$. The power-law slopes obtained by this fitting are listed in Table III. ${ }^{42}$ For all cases $m_{\text {eff }}>m$, having a magnitude nearly equivalent to

TABLE III

SCALING EXPONENTS AND INTERMOLECULAR REPULSIVE POTENTIAL PARAMETERS FOR LJ PARTICLES

\begin{tabular}{lrcc}
\hline $3 \gamma$ & $m$ & $m_{\text {eff }}$ & $m_{\text {IPL }}$ \\
\hline $10.5 \pm 0.2$ & 8 & 10.9 & $11.0 \pm 0.01$ \\
$15.0 \pm 0.2$ & 12 & 14.9 & $15.3 \pm 0.01$ \\
$29.3 \pm 0.3$ & 24 & 27.2 & $27.2 \pm 0.03$ \\
$40.2 \pm 0.6$ & 36 & 39.9 & $39.4 \pm 0.03$ \\
\hline
\end{tabular}


the corresponding value of $3 \gamma$. This supports a connection between density scaling and the IPL approximation to the intermolecular potential.

\section{FLUCTUATIONS OF THE POTENTIAL ENERGY AND THE VIRIAL}

There is an additional means to test the reliability of the IPL approximation and its connection to density scaling. If Eq. 6 applies, a relationship exists between $U(r)$ and the virial pressure, $W$. The virial pressure is the configurational part of the pressure (and hence, $W=0$ for an ideal gas), defined as

$$
W=-\frac{1}{3} r \frac{d U}{d r}
$$

If $U$ is a simple power law, substituting into Eq. 9 yields

$$
W=\frac{m_{\mathrm{IPL}}}{3} U
$$

Or for equilibrium fluctuations of the potential energy and the virial pressure s8-60 $^{58}$

$$
\frac{\Delta W}{\Delta U}=\frac{m_{\mathrm{IPL}}}{3}
$$

where $\Delta W=\frac{W-\langle W\rangle}{\sqrt{\left\langle\Delta W^{2}\right\rangle}}$ and $\Delta U=\frac{U-\langle U\rangle}{\sqrt{\left\langle\Delta U^{2}\right\rangle}}$, the brackets signifying average values. This predicted correlation of fluctuations in $W$ and $U$ is illustrated in Figure 9 for an LJ fluid with $m=8 .{ }^{60}$ From the slope of the linear fit of $\Delta W$ to $\Delta U, m_{\mathrm{IPL}}=11.0$ is obtained, which is equivalent to the effective slope of $U(r), m_{\mathrm{eff}}=10.9$ and not far from the value of $3 \gamma=10.5$ obtained by scaling the diffusion constant. Results for the four LJ liquids are included in Table III. ${ }^{60}$ The good agreement of $\gamma$ with the repulsive potential's dependence on particle separation, as quantified from either $m_{\mathrm{IPL}}$ or $m_{\mathrm{eff}}$, corroborates the underlying basis for density scaling. Scaling exponents for real liquids and polymers thus provide a measure of the forces between molecules in dense fluids and polymers.

\section{COMPARISON TO TIME-TEMPERATURE SUPERPOSITIONING}

Time-temperature superpositioning, in which master curves are constructed from measurements covering a limited range of frequencies at various temperatures, ${ }^{21,61,62}$ is a popular method of extending the dynamic range of experimental data. The main requirement for its application is that the molecular motions relevant to the property of interest are the same at all test temperatures or otherwise all motions have the same temperature dependence. The original idea underlying the time-temperature superposition principle is that the rates of molecular and chain motions are governed by the free (unoccupied) volume. This idea is likely incorrect, as indicated by values of $E_{\mathrm{\rho}} / E_{P}$ in Table I that are significantly larger than zero. Nevertheless, polymers often behave thermorheologically simple, whereby $t$ - $T$ superpositioning can yield accurate master curves, as affirmed by the many examples in the literature. ${ }^{14,21}$

There are two limitation of $t-T$ superpositioning. First, if data for polymers extend into the softening zone of the viscoelastic response, the local segmental modes begin to contribute. These local modes are more sensitive to temperature than the global chain modes dominating the rubbery plateau and terminal zones; consequently, in the glass transition zone where both local and global modes contribute to the viscoelastic properties, polymers exhibit thermo-rheological complexity. ${ }^{14,63}$ This breakdown of time-temperature superpositioning was first discovered for polystyrene, ${ }^{64}$ which is often cited as the prototypical example of $t$-T equivalence! Equally ironic, 


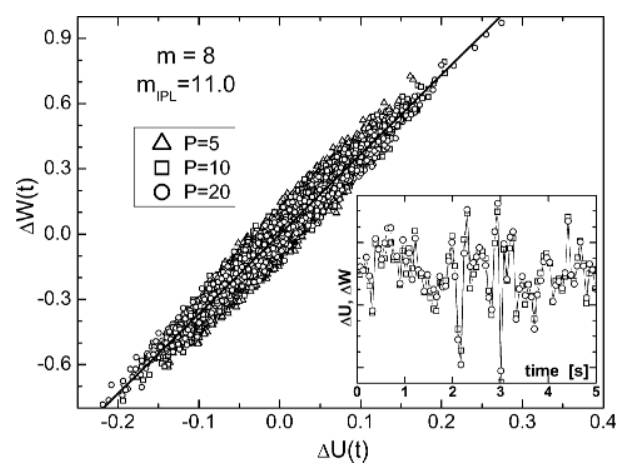

FIG. 9. - Fluctuations in the virial pressure versus fluctuation in the potential energy for LJ particles with $m=8$. The line through the data is the linear least squares fit, the slope of which is $m_{\mathrm{IPL}}=11$. The inset, showing resentative fluctuations in the two quantities over time, illustrates the correlations of the two quantities.

the most dramatic illustration of thermo-rheological complexity is polyisobutylene, ${ }^{65}$ the polymer largely responsible for the universal acceptance of the $t-T$ principle. $^{66}$

The second problem with superpositioning is that although it has been extended to pressure, ${ }^{21}$ the basis for obtaining master curves of pressure-shifted data is ill-founded, and examples of timepressure superpositioning are sparse. Moreover, $t-P$ superpositioning fails in the transition zone for the same reason $t-T$ superpositioning fails, as demonstrated, for example, for polyisoprene. ${ }^{67}$ Thus, density scaling supplements traditional time-temperature superpositioning by addressing in a fundamental way the effects of pressure and volume on the dynamics. However, unlike $t-T$ superpositioning, density scaling is valid for all thermodynamic conditions of rubbery polymers and liquids, from the deeply supercooled state to high temperatures at which Arrhenius behavior obtains. The disadvantage of density scaling is that its application requires knowledge of both the temperature and pressure dependences of the density.

\section{E. BOYER-SPENCER RULE}

An empirical "rule-of-thumb" for polymers is that the product of the thermal expansion coefficient, $\alpha_{P}=\left.\rho^{-1} \frac{d \rho^{-1}}{d T}\right|_{P}$, and the glass transition temperature is a

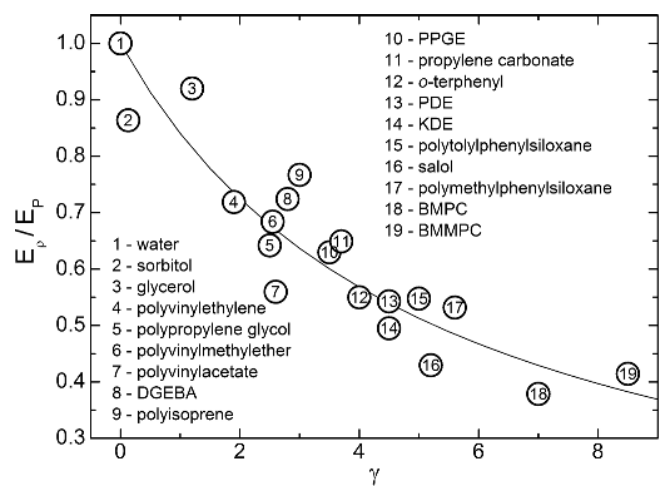

FIG. 10. - The relationship between the activation energy ratio and the scaling exponent. The solid line is the fit of Eq. 12, yielding $\alpha_{P} T_{g}=0.19 \pm 0.01$. The value of $\gamma$ for $\mathrm{H}$-bonded materials is approximate because density scaling breaks down due to the dependence of hydrogen bond concentration on state temperature and pressure. 
universal constant having a value of $\alpha_{P} T_{g} \sim 0.16 .^{68,69}$ From Eqs. 2, 3, and 8, the following relation can be derived ${ }^{37}$ :

$$
\frac{E_{\rho}}{E_{P}}=\frac{1}{1+\alpha_{P} T \gamma}
$$

If the product $\alpha_{P} T_{g}$ is indeed constant, then data for various materials of $E_{\mathrm{\rho}} / E_{P}$ versus $\gamma$ will fall on a single curve; that is, the scaling exponent uniquely defines the value of the activation energy ratio at $T_{g}$. Results for both polymers and molecular liquids are shown in Figure $10,{ }^{29}$ with the data well described by Eq. 12 using $\alpha_{P} T_{g}=0.19 \pm 0.01$, a value in accord with the empirical result. ${ }^{58,59}$ This agreement give further credence to the applicability of density scaling to experimental data for real materials.

\section{SUMMARY}

The respective influences of thermal contraction and the loss of thermal energy on the spectacular slowing down of molecular and segmental motions on approach to the glass transition can be assessed from the ratio of the isochoric and isobaric activation energies. This ratio is close to 0.5 for molecular liquids but is larger for polymers, signifying the greater effectiveness of thermal energy than density in controlling the relaxation properties of the latter. Temperature tends to be the stronger control parameter for chain molecules due to the negligible effect of pressure on intramolecular bonds. Relaxation times, diffusion constants, and viscosities depend uniquely on the ratio of temperature to density raised to the power of $\gamma$, with the exponent a material constant. From molecular dynamics simulations, this scaling exponent is found to have a magnitude governed by the intermolecular repulsive potential. The latter also governs the correlation between fluctuations in the potential energy and virial pressure. These results are strictly true only for an IPL potential but evidently well approximated by real materials, in which, however, the steepness of the repulsive potential is affected indirectly by the attractive forces.

\section{ACKNOWLEDGEMENTS}

I thank many collaborators, in particular R. Casalini, D. Coslovich, D. Fragiadakis, K. L. Ngai, M. Paluch, C. G. Robertson, and P. G. Santangelo. The financial support of the Office of Naval Research is greatly appreciated.

\section{REFERENCES}

${ }^{1}$ G. Heinrich, RubBer CHEM. TEChNOL. 70, 1 (1997).

${ }^{2}$ M. Kluppel and G. Heinrich, RubBer CHEM. TECHNOL. 73, 578 (2000).

${ }^{3}$ B. N. J. Persson, U. Tartaglino, O. Albohr, and E. Tosatti, Phys. Rev. B 71, 035428 (2005).

${ }^{4}$ R. Bond, G. F. Morton, and L. H. Krol, Polymer 25, 132 (1984).

${ }^{5}$ R. R. Rahalkar, RubBER CHEM. TECHNOL. 62, 246 (1989).

${ }^{6}$ H. Takino, R. Nakayama, Y. Yamada, S. Kohjiya, and T. Matsuo, RubBER ChEM. TeChnOL. 70, 584 (1997).

${ }^{7}$ G. Heinrich and H. B. Dumler, RuBBER CHEM. TECHNOL. 71, 53 (1998).

${ }^{8}$ R. N. Capps, Elastomeric Materials for Acoustical Applications, Naval Research Laboratory, Orlando, FL (1989).

${ }^{9}$ Sound and Vibration Damping with Polymers, R. D. Corsaro and L. H. Sperling, Eds., ACS Symposium Series 424,

American Chemical Society, Washington, DC, 1990.

${ }^{10}$ J. M. Gosline, RubBER CHEM. TECHNOL. 60, 417 (1987).

${ }^{11}$ P. Toensmeier, Defense Tech. Int. Sept./Oct., 18 (2005). 
${ }^{12}$ R. Bogoslovov, C. M. Roland, and R. M. Gamache, Appl. Phys. Lett. 90, 221910 (2007).

${ }^{13}$ C. M. Roland, D. Fragiadakis, and R. M. Gamache, Comp. Structures 92, 1059 (2010).

${ }^{14}$ C. M. Roland, Viscoelastic Behavior of Rubbery Materials, Oxford University Press (2011).

${ }^{15}$ C. M. Roland, Macromolecules 43, 7875 (2010).

${ }^{16}$ R. B. Bogoslovov, T. E. Hogan, and C. M. Roland, Macromolecules 43, 2904 (2010).

${ }^{17}$ M. Goldstein, J. Chem. Phys. 51, 3728 (1969).

${ }^{18}$ C. A. Angell, Science 267, 1924 (1995).

${ }^{19}$ S. Sastry, Nature 409, 164 (2001).

${ }^{20}$ P. B. Macedo and T. A. Litovitz, J. Chem. Phys. 42, 245 (1965).

${ }^{21}$ J. D. Ferry, Viscoelastic Properties of Polymers, 3rd ed., Wiley, New York (1980).

${ }^{22}$ G. S. Grest and M. H. Cohen, Adv. Chem. Phys. 48, 455 (1981).

${ }^{23}$ T. Pakula, J. Molec. Liq. 86, 109 (2000).

${ }^{24}$ J. T. Bendler, J. J. Fontanella, and M. Schlesinger, Phys. Rev. Lett. 87, 195503 (2001).

${ }^{25}$ D. Fragiadakis, R. Casalini, R. B. Bogoslovov, C. G. Robertson, and C. M. Roland, Macromolecules 44, 1149 (2011).

${ }^{26} \mathrm{G}$. Floudas and T. Reisinger, J. Chem. Phys. 111, 5201 (1999).

${ }^{27}$ G. Williams, Trans. Faraday Soc. 60, 1548 (1964); 61, 1564 (1965).

${ }^{28}$ P. Ortiz-Serna, R. Diaz-Calleja, M. J. Sanchis, G. Floudas, R. C. Nunes, A. F. Martins, and L. L. Visconte, Macromolecules 43, 5094 (2010).

${ }^{29}$ C. M. Roland, S. Hensel-Bielowka, M. Paluch, and R. Casalini, Rep. Prog. Phys. 68, 1405 (2005).

${ }^{30}$ C. M. Roland, K. J. McGrath, and R. Casalini, J. Non-Cryst. Sol. 352, 4910 (2006).

${ }^{31}$ C. M. Roland, T. Psurek, S. Pawlus, and M. Paluch, J. Polym. Sci. Polym. Phys. Ed. 41, 3047 (2003).

${ }^{32}$ R. Casalini and C. M. Roland, Macromolecules 38, 1779 (2005).

${ }^{33}$ C. M. Roland, R. Casalini, R. Bergman, and J. Mattsson, Phys. Rev. B 77, 012201 (2008).

${ }^{34}$ M. Naoki and S. Katahira, J. Phys. Chem. 95, 431 (1991).

${ }^{35}$ P. H. Poole, F. Sciortino, T. Grande, H. E. Stanley, and C. A. Angell, Phys. Rev. Lett. 73, 1632 (1994).

${ }^{36}$ R. E. Cook, H. E. King, and D. G. Peiffer, Phys. Rev. Lett. 69, 3072 (1992).

${ }^{37}$ R. Casalini and C. M. Roland, Phys. Rev. E 69, 062501 (2004).

${ }^{38}$ J. P. Hansen, Phys. Rev. A 2, 221 (1970).

${ }^{39}$ R. Agrawal and D. A. Kofke, Phys. Rev. Lett. 74, 122 (1995).

${ }^{40}$ W. G. Hoover and M. Ross, Contemp. Phys. 12, 339 (1971).

${ }^{41}$ N. H. March and M. P. Tosi, Introduction to the Liquid State, World Scientific (2002).

${ }^{42}$ D. Coslovich and C. M. Roland, J. Phys. Chem. B 112, 1329 (2008).

${ }^{43}$ J. E. Jones, Proc. Roy. Soc. Lond. A 106, 441 (1924); 106, 462 (1924).

${ }^{44}$ J. F. Dobson, K. McLennan, A. Rubio, J. Wang, T. Gould, H. M. Le, and B. P. Dinte, Aust. J. Chem. 54, 513 (2001).

${ }^{45}$ E. A. Moelwyn-Hughes, Physical Chemistry, 2nd ed., Pergamon Press, New York (1961).

${ }^{46}$ V. Y. Bardik and V. M. Sysoev, Low Temp. Phys. 24, 601 (1998).

${ }^{47}$ B. Widen, Science 157, 375 (1967).

${ }^{48}$ D. Chandler, J. D. Weeks, and H. C. Andersen, Science 220, 787 (1983).

${ }^{49}$ H. Stillinger, P. G. Debenedetti, and T. M. Truskett, J. Phys. Chem. B 105, 11809 (2001).

${ }^{50}$ T. B. Schrder, N. P. Bailey, U. R. Pedersen, N. Gnan, and J. C. Dyre, J. Chem. Phys. 131, 234503 (2009).

${ }^{51}$ J. D. Weeks, D. Chandler, and H. C. Andersen, J. Chem. Phys. 54, 5237 (1971).

${ }^{52}$ A. Tölle, H. Schober, J. Wuttke, O. G. Randl, and F. Fujara, Phys. Rev. Lett. 80, 2374 (1998).

${ }^{53}$ B. B. Laird and A. D. J. Haymet, Mol. Phys. 75, 71 (1992).

${ }^{54}$ Y. Hiwatari, H. Matsuda, T. Ogawa, N. Ogita, and A. Ueda, Prog. Theor. Phys. 52, 1105 (1974).

${ }^{55}$ W. T. Ashurst and W. G. Hoover, Phys. Rev. A 11, 658 (1975). 
${ }^{56}$ D. Fragiadakis and C. M. Roland, J. Chem. Phys. 134, 044504 (2011).

${ }^{57}$ C. M. Roland, S. Bair, and R. Casalini, J. Chem. Phys. 125, 124508 (2006).

${ }^{58}$ N. P. Bailey, U. R. Pedersen, N. Gnan, T. B. Schrder, and J. C. Dyre, J. Chem. Phys. 129, 184507 (2008).

${ }^{59}$ U. R. Pedersen, N. P. Bailey, T. B. Schrder, and J. C. Dyre, Phys. Rev. Lett. 100, 015701 (2008).

${ }^{60}$ D. Coslovich and C. M. Roland, J. Chem. Phys. 130, 014508 (2009).

${ }^{61}$ A. V. Tobolsky and R. D. Andrews, J. Chem. Phys. 13, 3 (1945).

${ }^{62}$ E. R. Fitzgerald, L. D. Grandine, and J. D. Ferry, J. Appl. Phys. 24, 650 (1953); J. D. Ferry, L. D. Grandine, and E. R. Fitzgerald, J. Appl. Phys. 24, 911 (1953).

${ }^{63}$ K. L. Ngai and D. J. Plazek, RubBer CheM. TeChNOL. 68, 376 (1995).

${ }^{64}$ D. J. Plazek, J. Phys. Chem. 69, 3480 (1965).

${ }^{65}$ D. J. Plazek, I.-C. Chay, K. L. Ngai, and C. M. Roland, Macromolecules 28, 6432 (1995).

${ }^{66}$ D. J. Plazek, Rheology. 40, 987 (1996).

${ }^{67}$ G. Floudas, C. Gravalides, T. Reisinger, and G. J. Wegner, J. Chem. Phys. 111, 9847 (1999).

${ }^{68}$ R. F. Boyer and R. S. Spencer, J. Appl. Phys. 15, 398 (1944).

${ }^{69}$ D. W. Van Krevelen, Properties of Polymers, Elsevier (1990).

[Received March 2012, Revised April 2012] 